\title{
Mycoplasma hominis brain abscess presenting after a head trauma: a case report
}

\author{
Andrés F Henao-Martínez ${ }^{1 *}$, Heather Young ${ }^{1}$, Johanna Jacoba Loes Nardi-Korver ${ }^{2}$ and William Burman
}

\begin{abstract}
Introduction: Mycoplasma hominis brain abscess is a rare occurrence, and treatment is not well defined. The mechanism by which M. hominis infects sites outside the genitourinary tract, including the central nervous system, is unclear.

Case presentation: We report the case of a 40-year-old Somali man who sustained a traumatic brain injury that required initial neurosurgical hematoma evacuation and that subsequently was complicated by a hospital-acquired M. hominis brain abscess. Our patient was successfully treated with neurosurgical debridement and an antibiotic course of intravenous doxycycline.
\end{abstract}

Conclusions: Head trauma or neurosurgical procedures or both might be a predisposing factor for this type of infection.

\section{Introduction}

Mycoplasma hominis is generally a genitourinary (GU) pathogen. M. hominis brain abscess is rare. To the best of our knowledge, only ten cases have been reported in the literature. However, the true incidence may be higher than this because the organism is difficult to grow in vitro. Optimal treatment of $M$. hominis brain abscess is not well defined in the literature. The organism is uniformly resistant to macrolides, and other potentially useful agents do not penetrate the central nervous system well.

\section{Case presentation}

A previously healthy 40-year-old Somali man presented after a motor vehicle accident. Injuries included a right subdural hematoma, subarachnoid hemorrhages, intraparenchymal contusions, facial fractures, and a left humerus fracture. The subdural hematoma was evacuated by means of a craniotomy on hospital day 7 . On hospital day 13, our patient developed a fever of $39.2{ }^{\circ} \mathrm{C}$ and purulent drainage from the craniotomy wound. A computed tomography $(\mathrm{CT})$ scan of his head without intravenous contrast demonstrated multiple foci of gas-containing abscesses

\footnotetext{
* Correspondence: andres.henaomartinez@ucdenver.edu

${ }^{1}$ Department of Medicine, Division of Infectious Diseases, 12700 E. 19th

Avenue, Mail Stop B168, Aurora, CO 80045, USA

Full list of author information is available at the end of the article
}

along the margin of the craniotomy (Figure 1a). The air in the abscess was considered part of the expected postcraniotomy changes. Incision, drainage, and removal of the bone flap were promptly performed. Vancomycin and piperacillin/tazobactam were initiated and then narrowed to ceftriaxone and metronidazole when a Gram stain revealed many polymononuclear cells but no organisms. Despite this management, fevers continued and our patient's mental status failed to improve. A CT scan of his head without intravenous contrast on hospital day 20 revealed an increase in both the size and extent of the brain abscess (Figure 1a). Our patient underwent a second debridement; again a Gram stain revealed no organisms. A pathology examination of the brain abscess showed a cerebral abscess in the superior temporal lobe with necrosis but without organisms (Figure 1d).

Cultures on sheep's blood agar from the neurosurgical debridements revealed small colonies with a peripheral clearing ('fried egg' morphology). Through sequencing of the $16 \mathrm{~S}$ ribosomal subunit region at a reference laboratory, the isolate was identified as M. hominis with $100 \%$ homology to reference strains. Our patient's antibiotics were changed to doxycycline $100 \mathrm{mg}$ intravenously twice per day on hospital day 24. His mental status and fevers improved significantly. The abscess had almost fully resolved by hospital day 40 (Figure 1c).

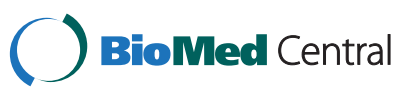




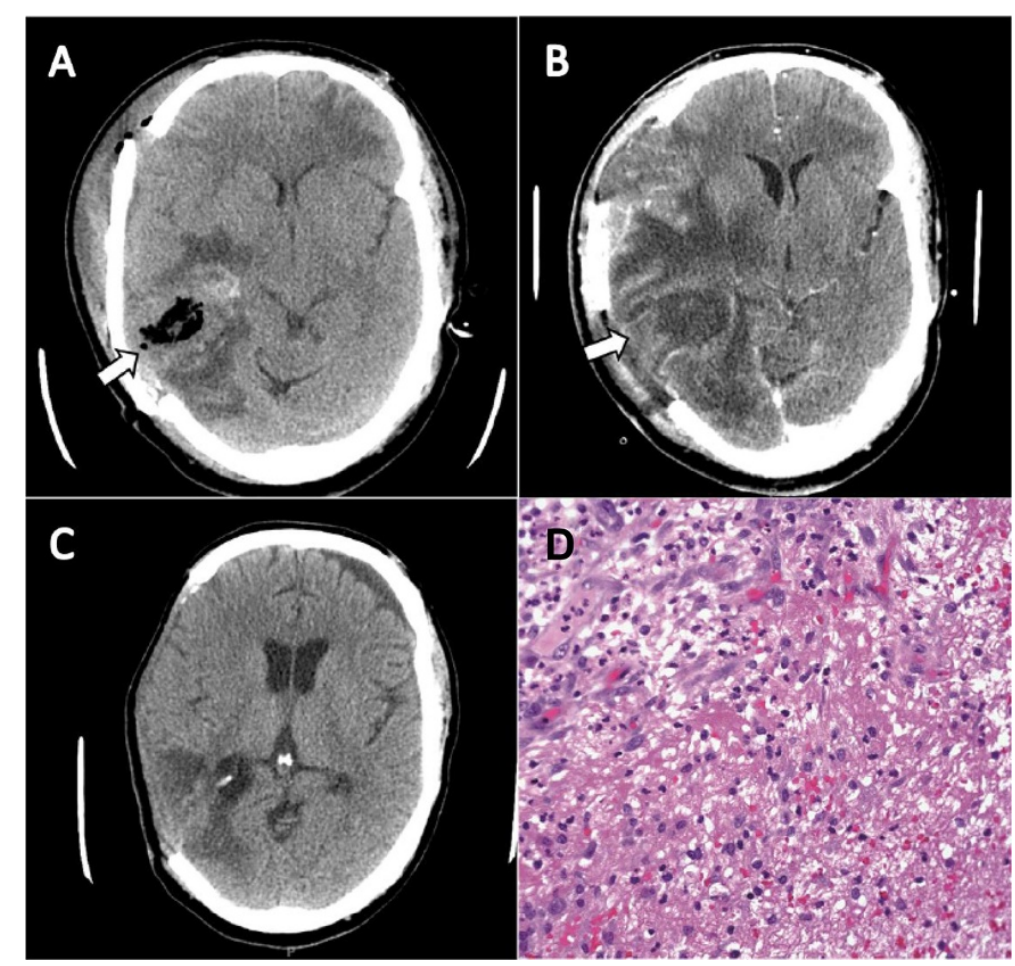

Figure 1 CT images and Pathology description: (a) Multiple foci of gas-containing abscesses along the margin of the craniotomy (arrow). (b) Significant increase in the size and extent of the brain abscess (arrow). (c) Resolving brain swelling and abscess. (d) Cerebral abscess in the superior temporal lobe with necrosis, newly formed capillaries, and scattered fibroblasts. The inflammatory infiltrate includes neutrophils, lymphocytes, plasma cells, and histiocytes (stain: hematoxylin and eosin; magnification: $\times 400$ ).

\section{Discussion}

M. hominis is traditionally considered to be a GU pathogen and has been implicated in non-gonococcal urethritis and post-partum fevers and is a possible cause of bacterial vaginosis [1,2]. In addition to causing infection, $M$. hominis is well known to colonize the $\mathrm{GU}$ and respiratory tracts [3-5]. M. hominis infections outside the GU tract are uncommon, but the organism has been isolated in cases of bacteremia, inguinal wound infections, sternotomy infection, septic prosthetic hip arthritis, pleural empyema, ventriculo-peritoneal shunt infections, and postneurosurgical wound infections [6].

Eleven cases of M. hominis brain abscess [7-15], including this case, have been published (Table 1). Eight of these cases were related to head trauma or craniotomy. The mean time to $M$. hominis isolation in the published literature is 13.5 days, suggesting that patients acquire M. hominis brain abscess while in the hospital. Hemorrhagic lesions in the brain could be seeded by contiguous infection after disruption of a colonized upper airway following

Table 1 Cases of adult Mycoplasma hominis brain abscess

\begin{tabular}{llllll}
\hline Case & Year & Age and sex & Associated condition & First culture after AD & Therapy \\
\hline 1 & 1981 & 29-year-old man & Head trauma & 21 days & Tetracycline \\
\hline 2 & 1995 & 20-year-old man & Head trauma & 14 days & Cefotaxime + metronidazole + doxycycline \\
\hline 3 & 1997 & 22-year-old woman & Post-partum & 8 days & Ceftriaxone + metronidazole \\
\hline 5 & 2002 & 40-year-old woman & Cavernous angioma/craniotomy & 4 days & Ciprofloxacin + metronidazole \\
\hline 6 & 2003 & 17-year-old girl & Post-partum & 13 days & Doxycycline + clindamycin \\
\hline 7 & 2004 & 40-year-old man & Head trauma & 14 days & Tetracycline \\
\hline 8 & 2008 & 17-year-old girl & Head trauma & 17 days & Gatifloxacin \\
\hline 9 & $2003 / 2006$ & NA & Craniotomy for colloid cyst removal & 18 days & Gatifloxacin + clindamycin \\
\hline $10^{\text {a }}$ & 2011 & 40-year-old man & Head trauma & NA & Doxycycline \\
\hline
\end{tabular}

apresent report. $A D$ : admission day; $N A$ : not available. 
trauma or after transient bacteremia from manipulation of the colonized oropharyngeal or GU tracts or both.

$M$. hominis is uniformly resistant to macrolides. Fluoroquinolones, tetracyclines, and clindamycin have better in vitro antimicrobial activity against $M$. hominis [16,17]. These three classes of antibiotics possess similar, moderate rates of blood-brain barrier penetration [18]. Rates of in vitro resistance to these antibiotics may be as high as $10 \%$ for clindamycin, $27 \%$ for doxycycline, and $80 \%$ for ciprofloxacin. However, there have been successful reports for each of these therapies.

\section{Conclusions}

$M$. hominis brain abscess is rare. The mechanism of $M$. hominis brain abscess is likely to be either direct spread from a colonized oropharynx to an open head wound or seeding of an intra-cranial hematoma from transient bacteremia after manipulation of the GU tract. $M$. hominis brain abscesses are treatable with surgical drainage and appropriate antimicrobial therapy of a tetracycline, clindamycin, or fluoroquinolone.

\section{Consent}

Written informed consent was obtained from the patient for publication of this case report and accompanying images. A copy of the written consent is available for review by the Editor-in-Chief of this journal.

\section{Abbreviations \\ CT: computed tomography; GU: genitourinary.}

\section{Competing interests}

The authors declare that they have no competing interests.

\section{Authors' contributions}

AFH-M was the major contributor in studying the case and writing the manuscript and was involved in the medical care of the patient. HY contributed to the writing and editing of the manuscript. JJLN-K is on the faculty of the Department of Pathology and was responsible for the brain biopsy reading. WB is on the faculty of the Division of Infectious Diseases, is the director of Denver Public Health, and was involved in the manuscript editing and in the medical care of the patient. All authors read and approved the final manuscript.

\section{Acknowledgments}

No funding agencies had a role in the preparation, review, or approval of the manuscript. The views expressed in this article are those of the authors and do not necessarily represent the views of the University of Colorado Denver.

\section{Author details}

${ }^{1}$ Department of Medicine, Division of Infectious Diseases, 12700 E. 19th Avenue, Mail Stop B168, Aurora, CO 80045, USA. ²Department of Pathology, University of Colorado Denver, 12700 E. 19th Avenue, Mail Stop B168, Aurora, CO 80045, USA.

Received: 20 March 2012 Accepted: 8 June 2012

Published: 22 August 2012

\section{References}

1. Kilic D, Basar MM, Kaygusuz S, Yilmaz E, Basar H, Batislam E: Prevalence and treatment of Chlamydia trachomatis, Ureaplasma urealyticum, and Mycoplasma hominis in patients with non-gonococcal urethritis. Jpn J Infect Dis 2004, 57:17-20.
2. Germain M, Krohn MA, Hillier SL, Eschenbach DA: Genital flora in pregnancy and its association with intrauterine growth retardation. J Clin Microbiol 1994, 32:2162-2168

3. Clegg A, Passey M, Yoannes M, Michael A: High rates of genital mycoplasma infection in the highlands of Papua New Guinea determined both by culture and by a commercial detection kit. J Clin Microbiol 1997, 35:197-200

4. Mufson MA: Mycoplasma hominis: a review of its role as a respiratory tract pathogen of humans. Sex Transm Infect 1983, 10:335-340.

5. Lusk MJ, Konecny P, Naing ZW, Garden FL, Cumming RG, Rawlinson WD: Mycoplasma genitalium is associated with cervicitis and HIV infection in an urban Australian STI clinic population. Sex Transm Infect 2011, 87:107-109.

6. Madoff S, Hooper DC: Nongenitourinary infections caused by Mycoplasma hominis in adults. Rev Infect Dis 1988, 10:602-613.

7. Payan DG, Seigal N, Madoff S: Infection of a brain abscess of Mycoplasma hominis. J Clin Microbiol 1981, 14:571-573.

8. Kersten RC, Haglund L, Kulwin DR, Ma'luf R, DeConciliis C: Mycoplasma hominis orbital abscess. Arch Ophthalmol 1995, 113:1096-1097.

9. Zheng X, Olson DA, Tully JG, Watson HL, Cassell GH, Gustafson DR, Svien KA, Smith TF: Isolation of Mycoplasma hominis from a brain abscess. J Clin Microbiol 1997, 35:992-994.

10. House P, Dunn J, Carroll K, MacDonald J: Seeding of a cavernous angioma with Mycoplasma hominis: case report. Neurosurgery 2003, 53:749-752. discussion 752-743.

11. Douglas MW, Fisher DA, Lum GD, Roy J: Mycoplasma hominis infection of a subdural haematoma in the peripartum period. Pathology 2003, 35:452-454.

12. Kupila L, Rantakokko-Jalava K, Jalava J, Peltonen R, Marttila RJ, Kotilainen E, Kotilainen P: Brain abscess caused by Mycoplasma hominis: a clinically recognizable entity? Eur J Neurol 2006, 13:550-551.

13. McCarthy KL, Looke DF: Successful treatment of post-neurosurgical intracranial Mycoplasma hominis infection using gatifloxacin. J Infect. 2008, 57:344-346.

14. Kupila L, Rantakokko-Jalava K, Jalava J, Nikkari S, Peltonen R, Meurman O, Marttila RJ, Kotilainen E, Kotilainen P: Aetiological diagnosis of brain abscesses and spinal infections: application of broad range bacterial polymerase chain reaction analysis. J Neurol Neurosurg Psychiatry 2003, 74:728-733.

15. Al Masalma M, Armougom F, Scheld WM, Dufour H, Roche PH, Drancourt M, Raoult D: The expansion of the microbiological spectrum of brain abscesses with use of multiple $16 \mathrm{~S}$ ribosomal DNA sequencing. Clin Infect Dis 2009, 48:1169-1178.

16. Krausse R, Schubert S: In-vitro activities of tetracyclines, macrolides, fluoroquinolones and clindamycin against Mycoplasma hominis and Ureaplasma ssp. isolated in Germany over 20 years. Clin Microbiol Infect 2010, 16:1649-1655.

17. McCormack WM: Susceptibility of mycoplasmas to antimicrobial agents: clinical implications. Clin Infect Dis 1993, 17(Suppl 1):S200-\$201.

18. Nau R, Sorgel F, Eiffert $H$ : Penetration of drugs through the bloodcerebrospinal fluid/blood-brain barrier for treatment of central nervous system infections. Clin Microbiol Rev 2010, 23:858-883.

doi:10.1186/1752-1947-6-253

Cite this article as: Henao-Martínez et al:: Mycoplasma hominis brain abscess presenting after a head trauma: a case report. Journal of Medical Case Reports 2012 6:253. 